\title{
On the group ring of a finite abelian group
}

\section{Raymond G. Ayoub and Christine Ayoub}

\begin{abstract}
The group ring of a finite abelian group $G$ over the field of rational numbers $Q$ and over the rational integers $Z$ is studied. A new proof of the fact that the group ring $Q G$ is a direct sum of cyclotomic fields is given - without use of the Maschke and Wedderburn theorems; it is shown that the projections of $Q G$ onto these fields are determined by the inequivalent characters of $G$. It is proved that the group of units of $Z G$ is a direct product of a finite group and a free abelian group $F$ and the rank of $F$ is determined. A formula for the orthogonal idempotents of $Q G$ is found.
\end{abstract}

\section{Introduction}

In this paper we study the group ring of a finite abelian group $G$ over the field $Q$ of rational numbers and over the (rational) integers $Z$. We give a new proof of the well-known fact that the group ring $Q G$ is a direct sum of cyclotomic fields [G. Higman] which enables us to say which fields arise and with what multiplicity (see also [5]). We show that the projections of $Q G$ onto these fields are determined by the inequivalent characters of $G$. This proof is to our mind conceptually much simpler then the one using representation theory. Granted an acquaintance with the elementary properties of tensor products (of commutative algebras) all that is needed is developed in the paper itself.

In $\$ 3$ we consider the group $U$ of units of $Z G$; we find that $U$ is the direct product of a finite group and a free abelian group $F$ of finite rank - a fact already established by Higman (cf. [4]) - but our results

Received 21 March 1969. Received by J.. Austral. Math. Soc. 17 September 1968. Revised 17 December 1968. Communicated by G.B. Preston. This research was done under NSF Contract GP-5593. 
enable us to calculate the rank of the group $F$ (Theorem 4). Theorem 5 gives a formula for the orthogonal idempotents of $Q G$. Finally in the last paragraph we study the embedding of $Z G$ in the direct sum of cyclotomic fields, in the case when $G$ is cyclic of prime power order.

\section{Definitions and notations}

We list the notations which will be used throughout the paper. Some of these are standard, while others are our own invention - most of the latter will be explained in the body of the paper but are listed here for the convenience of the reader.

$\oplus$ is used for the direct sum of rings.

If $X_{i}$ are rings and if $x_{i} \in X_{i}$ (for $i \in I$ ), we write $\underset{i \in I}{\oplus x_{i}}$ for the element of $\underset{i \in I}{\oplus} X_{i}$ whose $i$-th component is $x_{i}$.

$\otimes$ denotes the tensor product over the rational field.

If $R$ is a ring, we write $m R$ for $R \oplus \ldots \oplus R$ ( $m$ terms) and $R^{m}$ for $R \otimes \ldots \otimes R \quad(m$ terms $)$.

$Q$ is the field of rational numbers.

$\zeta_{d}$ denotes a primitive (complex) d-th root of unity. $\Phi_{d}(x)$ denotes the cyclotomic polynomial satisfied by the primitive $d$-th roots of unity over the rationals.

$Q_{d}$ is the splitting field of $\Phi_{d}(x)$, so that $Q_{d}$ is obtained from $Q$ by adjunction of $\zeta_{d}$.

$F[x]$ denotes the ring of the polynomials in $x$ over the field $F$ and $F(\alpha)$ the field obtained by adjunction of $\alpha$ to $F$.

If $f(x) \in F[x], \operatorname{deg}(f(x))=$ degree of $f(x),(f(x))=$ ideal generated by $f(x)$.

The exponent of a finite group $G$ is the least positive integer. $m$ such that $g^{m}=1$ for all $g \in G$. (a) is the cyclic group generated by $a$, and $o(a)=$ order of $a$.

$x$ denotes the direct product of multiplicative groups. 
We write our mappings on the left so that $\alpha \circ \beta$ denotes the mapping $\beta$ followed by the mapping $\alpha$.

For the homomorphism $n, \operatorname{Ker}(\eta)=$ kernel of $n$ and $(n \mid G)=$ restriction of $n$ to $G(G$ a group $)$.

$N$ and $Z$ denote the natural numbers and integers, respectively.

$Z_{d}$ denotes the polynomials in $\zeta_{d}$ over $Z$.

$\operatorname{Mod}_{2}\{S\}=$ module generated by $S$ over $Z$.

$Q G$ and $Z G$ are the group rings of $G$ over $Q$ and $Z$, respectively.

Actually only finite abelian groups are considered - but this is explicitly stated in the theorems.

\section{The structure of $Q G$}

DEFINITION 1. If $\zeta_{d}$ is a (complex) primitive $d$-th root of unity, $\left.Q_{d}=Q_{\left(\zeta_{d}\right.}\right)$. We note that $Q_{d}$ does not depend on the particular $d$-th root of unity chosen.

PROPOSITION 1. Let $G$ be a cyclic group of order $n$, and $Q$ the field of rational numbers. Then

$$
Q G \simeq \stackrel{\oplus}{Q} Q_{d}
$$

Proof. Let $\Phi_{d}(x)$ be the cyclotomic polynomial satisfied by the primitive $d-t h$ roots of unity over $Q$. Then

$$
\begin{aligned}
Q G & \simeq Q[x] /\left(x^{n}-1\right) \\
& \simeq \oplus{ }_{d \mid n} Q[x] /\left(\Phi_{d}(x)\right)
\end{aligned}
$$

But $Q[x] /\left(\Phi_{d}(x) \dot{\prime} \simeq Q\left(\zeta_{d}\right)=Q_{d}\right.$. Hence (1) $Q G \simeq \underset{d \mid n}{\oplus}{ }^{Q} d$.

Note. If $G$ is generated by $a$, then under the isomorphism (1),

$$
a^{i} \leftrightarrow \underset{d \mid n}{\oplus} \zeta_{d}^{i} \in \underset{d \mid n}{\oplus} Q_{d} \text {. }
$$


PROPOSITION 2. If the groups $A_{i}(1 \leqq i \leqq k)$ are subgroups of the group $G$ such that $G=A_{1} \times \ldots \times A_{k}$ (direct product) then

$$
Q G \simeq Q A_{1} \otimes \ldots \otimes Q A_{k} \text { (tensor product over Q). }
$$

Note. If $Z$ denotes the integers then we also have $Z G \simeq Z A_{1} \otimes \ldots \otimes Z A_{k}$. The proof follows in the same way.

Proof. If $g=a_{1} \ldots a_{k}$, where $a_{i} \in A_{i}(1 \leqq i \leqq k)$, map $g$ onto $a_{1} \otimes \ldots \otimes a_{k} \in Q A_{1} \otimes \ldots \otimes Q A_{k}$. This mapping sends a basis for $Q G$ onto a basis for $Q A_{1} \otimes \ldots \otimes Q A_{k}$ and hence we can extend it to an isomorphism.

PROPOSITION 3. $Q_{k} \otimes Q_{Z} \simeq$ direct sum of $\phi(d)$ copies of $Q_{m}=\phi(d) Q_{m}$, where $d=g . c \cdot d \cdot(k, Z), m=1 . c \cdot m \cdot(k, Z)$ and $\phi$ denotes the Euler $\phi$-function.

Proof. Let $m=k s=2 t$, where $(s, t)=1$. Then $\zeta_{m}^{s}$ and $\zeta_{m}^{t}$ are clearly primitive $k$-th and $l$-th roots of unity respectively. Thus the field $Q\left(\zeta_{k}, \zeta_{l}\right)$, obtained by adjoining to $Q$ primitive $k$-th and $l$-th roots of unity, is contained in $Q_{m}$; on the other hand, if we choose $u$ and $v$ such that $u s+v t=1$, then $\zeta_{m}=\left(\zeta_{m}^{s}\right)^{u}\left(\zeta_{m}^{t}\right)^{v}$ and hence $\zeta_{m}$ is in the field $Q\left(\zeta_{k}, \zeta_{l}\right)=Q_{k}\left(\zeta_{l}\right)$. Therefore, we have shown that

$$
Q_{m}=Q_{k}\left(\zeta_{l}\right)
$$

Now let

$$
\Phi_{l}(x)=f_{1}(x) \ldots f_{S}(x)
$$

be the decomposition of the cyclotomic polynomial into irreducible factors in $Q_{k}[x]$. Since by (4) $Q_{m}$ is obtained from $Q_{k}$ by adjoining any root of $\Phi_{2}(x)$ (and therefore, any root of any $f_{i}(x)$ ) $Q_{m} \simeq Q_{k}[x] /\left(f_{i}(x)\right)(1 \leqq i \leqq s)$. Thus each $f_{i}(x)$ has degree 


$$
\left[Q_{m}: Q_{k}\right]=\frac{\left[Q_{m}: Q\right]}{\left[Q_{k}: Q\right]}=\frac{\phi(m)}{\phi(k)}=\frac{\phi(z)}{\phi(d)}
$$

since $\phi(k) \phi(Z)=\phi(m) \phi(d)$. Also

$$
\phi(Z)=\operatorname{deg}\left(\Phi_{\eta}(x)\right)=s\left[\operatorname{deg}\left(f_{i}(x)\right)\right]=s \frac{\phi(Z)}{\phi(d)}
$$

so that

$$
s=\phi(d)
$$

From a known theorem we have that $Q_{k} \otimes Q_{2} \simeq Q_{k}[x] /\left(\Phi_{Z}(x)\right)$, and from the decomposition (5) and (6) this is isomorphic to

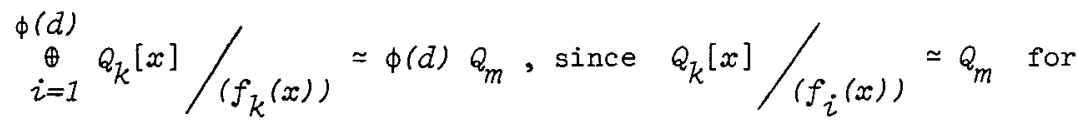

$1 \leqq i \leqq \phi(d)$.

Note. Applying Proposition 3 several times we could establish that for positive integers $k_{1}, \ldots, k_{r}$

$$
Q_{k_{1}} \otimes \cdots \otimes Q_{k_{p}} \simeq s Q_{m} \text {, }
$$

where $m=1 . c \cdot \mathrm{m} .\left(k_{1}, \ldots, k_{p}\right)$ and $s$ is a positive integer (which we could calculate). In particular, if g.c.d. $\left(k_{i}, k_{j}\right)=1$ for $i \neq j$,

$$
\left.Q_{k_{1}} \otimes \ldots \otimes Q_{k_{r}}=Q_{m} \text { (i.e. } s=1\right) \text {. }
$$

THEOREM 1. If $G$ is a finite abelian group of exponent $m$, there exist integers $u_{d} \geqq 0$ such that

$$
Q G \simeq \underset{d \mid m}{\oplus} u_{d} Q_{d}
$$

Proof. Let $G=A_{1} \times \ldots \times A_{k}$, where $A_{i}$ is cyclic of order $d_{i}$ for $1 \leqq i \leqq k$ and $d_{1}\left|d_{2}\right| \ldots \mid d_{k}=m$. Then by Proposition 2, $Q G \simeq Q A_{1} \otimes \ldots \otimes Q A_{k}$ and hence by Proposition 1, $Q G \simeq \underset{d \mid d_{1}}{\oplus} Q_{d} \otimes \ldots \underset{d \mid d_{k}}{\otimes} Q_{d}$. Now if we expand using the distributive law and the note after Proposition 3, we see that $Q G$ is the direct sum of 
cyclotomic fields $Q_{d}$, where each $d$ divides $m$.

\section{The projections of $Q G$}

DEFINITION 2. Let $G$ be a finite abelian group. The characters $X$ and $\psi$ are equivalent if $\operatorname{Ker}(X)=\operatorname{Ker}(\psi)$.

LEMMA 1. Let $G$ be a finite abelian group of order $n$ and exponent $m$; let $d$ divide $m$ and let $t_{d}$ be the number of cyclic subgroups of $G$ of order $d$. Then

(1) The number of inequivalent characters $x$ such that $\chi(G)=\left(\zeta_{d}\right)$ is $t_{d}$.

(2) If $\chi(G)=\left(\zeta_{d}\right)$, the number of characters equivalent to $x$ is $\phi(d)$.

(3) $\sum_{d \prod_{m}} t_{d} \phi(d)=n$.

Proof. (1) Let $D$ be a subgroup of $G$ such that $G / D$ is cyclic of order $d$, and let $\zeta_{d}$ be a primitive $d$-th root of unity. Then there exists an epimorphism from $G$ to $\left(\zeta_{d}\right)$ with kernel $D$-i.e. a character with kernel $D$. Therefore, the number of inequivalent characters $X$ such that $X(G)=\left(\zeta_{d}\right)$ is equal to the number of subgroups $D$ such that $G / D$ is cyclic of order $d$ and this is equal to the number of cyclic subgroups of order $d$.

(2) Let $\chi$ be a character with $\chi(G)=\left(\zeta_{d}\right)$ and $D=\operatorname{Ker}(\chi)$. Then for $\alpha$ in the automorphism group of $\left(\zeta_{d}\right) \psi_{\alpha}=\alpha \circ x$ is a character of $G$ with kernel $D$ - i.e. $\psi_{\alpha}$ is equivalent to $\chi$ - and distinct automorphisms a give rise to distinct characters $\psi_{\alpha}$. Furthermore, any character $\psi$ of $G$ which is equivalent to $\chi$ is of this form, since the mapping $\alpha$ defined by $\alpha(x(g))=\psi(g)$ for $g \in G$ is an automorphism of $\left(\zeta_{d}\right)$. But the automorphism group of $\left(\zeta_{d}\right)$ has order $\phi(d)$. Thus there are $\phi(d)$ characters equivalent to $x$.

(3) From (2) and (3) the total number of characters of $G$ is 
$\sum_{d \mid m} t_{d} \phi(d)$. Hence $n=\sum_{d \mid m} t_{d} \phi(d)$, since $G$ has $n$ distinct characters.

COROLLARY. If $x_{1}, \ldots, x_{2}$ are inequivalent characters of the finite abelian group $G$ of order $n$ and if $\sum_{i=1}^{l} \phi\left(d_{i}\right)=n$, where $x_{i}(G)=\left(\zeta_{d_{i}}\right)$ for $I \leqq i \leqq Z$, then the characters $x_{i}(1 \leqq i \leqq Z)$ form a cormplete set of inequivalent characters of $G$.

THEOREM 2. Let $G$ be a finite abelian group of exponent $m$. Then $Q G \simeq \oplus_{d \mid m}^{\oplus} t_{d} Q_{d}$, where $t_{d}$ is the number of cyclic subgroups of $G$ of order $d$. The projection $\pi$ of $Q G$ onto the component $Q_{d}$ defines a character $x=(\pi \mid G)$ of $G$ onto $\left(\zeta_{d}\right)$ and the characters so defined form a complete set of inequivalent characters of $G$.

Proof. From Theorem 1, we have

$$
Q G \simeq \underset{d \mid m}{\oplus} u_{d} Q_{d}
$$

It is clear that for each projection $\pi$ onto a component $Q_{d}$ we get a character $\chi=(\pi \mid G)$ of $G$. We show first that if $\pi_{1}$ and $\pi_{2}$ are projections onto different components then the characters $X=\left(\pi_{1} \mid G\right)$ and $\psi=\left(\pi_{2} \mid G\right)$ are not equivalent. For suppose to the contrary that $\psi$ is equivalent to $x$. Then if $\chi(G)=\left(\zeta_{d}\right)$ there is an automorphism $\alpha$ of $\left(\zeta_{d}\right)$ such that $\psi=\alpha \circ X$. Let $\bar{\alpha}$ be the automorphism of $Q_{d}$ which takes $\zeta_{d}$ into $\alpha\left(\zeta_{d}\right)$.

$$
\text { Since } Q G \simeq \underset{d \mid m}{\oplus} t_{d} Q_{d} \text {, there is an element } r \in Q G \text { with } \pi_{1}(r)=0
$$

and $\pi_{2}(r) \neq 0$. Now $r=\sum_{g \in G} r(g) g$ with $r(g) \in Q$ and we have:

$0=\pi_{1}(r)=\sum_{g \in G} r(G) \pi_{1}(g)=\sum_{g \in G} r(g) \times(g)$. On the other hand, 


$$
\begin{aligned}
0 \neq \pi_{2}(r)=\sum_{g \in G} r(g) \pi_{2}(g) & =\sum_{g \in G} r(g) \psi(g)=\sum_{g \in G} r(g)(\alpha \circ \chi)(g) \\
& =\bar{\alpha}\left[\sum_{g \in G} r(g) \chi(g)\right)=0 .
\end{aligned}
$$

This is clearly impossible so that $X$ and $\psi$ cannot be equivalent.

Let $\pi_{1}, \ldots, \pi_{l}$ be the projections of $Q G$ defined by (8), and let

$x_{i}=\left(\pi_{i} \mid G\right)$ for $1 \leqq i \leqq l$. We establish the fact that the $x_{i}$ form a complete set of inequivalent characters of $G$. Clearly if $\dot{\chi}_{i}(G)=\left(\zeta_{d_{i}}\right)$, $\pi_{i}(Q G)=Q_{d_{i}}$ since $G$ generates $Q G$ over $Q$. Hence $Q G \simeq \underset{i=1}{\oplus} Q_{i}$ so that comparing dimensions we have: $n=\sum_{i=1}^{l} \phi\left(d_{i}\right)$. Hence by the Corollary to Lemma 1 , the $\chi_{i}$ are a complete set of inequivalent characters of $G$. Thus also

$$
Q G \simeq \underset{d \mid m}{\oplus}{ }^{t} d Q_{d}
$$

REMARK. It may happen that $Q_{d}=Q_{d}$, with $d<d^{\prime}$ - in fact, this is the case if, and only if, $d$ is odd and $d^{\prime}=2 d$. It can be shown that the expression (8) (obtained by use of Propositions 1-3) is the same as (9) i.e. $u_{d}=t_{d}$. However, the proof is a little tedious and so we thought it not of sufficient interest to include.

\section{The units of $Z G$}

THEOREM 3. (G. Higman) The onzy units of finite order in $Z G$ are of the form $\pm g(g \in G)$.

Proof. Let $u \in Z G$ be a unit of finite order. Then if $\pi$ is the projection of $Q G$ in the isomorphism $Q G \simeq \underset{d \mid m}{\oplus} t_{d} Q_{d}, \pi(u)$ is a unit of finite order in $Q_{d}-i . e . \pi(u)$ is a root of unity. Thus if $u=\sum_{g \in G} u(g) g$ with $u(g) \in z, \pi(u)=\sum_{g \in G} u(g) \pi(g)=\sum_{g \in G} u(g) \chi(g)=\rho_{\chi}$, where $X=(\pi \mid G)$ and $\rho_{X}$ is a root of unity. Since these characters $X$ form a complete set of inequivalent characters we have: 


$$
\sum u(g) \chi(g)=\rho_{\chi} \text { for every } \chi \in \hat{G},
$$

the character group $G$ and where each $\rho_{X}$ is a root of unity. Using the orthogonality relations of the characters, we obtain $n u(g)=\sum_{x \in \hat{G}} \rho_{\chi} \overline{\chi(g)}$ for $g \in G$ and from this it is easy to deduce that there exists a $g_{0} \in G$ such that $u\left(g_{0}\right)= \pm 1$ and $u(g)=0$ for $g \neq g_{0}$ (cf.e.g. [1]). Thus $u= \pm g$.

THEOREM 4. Let $G$ be a finite abelian group of order $n$ and let $U$ denote the group of units of $Z G$. Then $U=T \times F$ with $T=\{ \pm g \mid g \in G\}$ and $E$ free abelian of rank $\frac{1}{2}\left(n+1+t_{2}-22\right)$, where $t_{2}=$ number of elements of $G$ of order 2 and $Z=$ number of cyclic subgroups of $G$.

Proof. $Z G$ is isomorphically embedded in $\underset{d \mid m}{\oplus} t_{d} Z_{d}$ under the isomorphism (9) (here $z_{d}=2\left(\zeta_{d}\right)$ ). Since both $2 G$ and $\oplus_{d \mid m}^{\oplus} t_{d} z_{d}$ are free abelian of rank $n, Z G$ is of finite index $k$ in $\underset{d \mid m}{\oplus} t_{d} z_{d}$

(identifying $Z G$ with its image under the isomorphism). Let $U$ denote the group of units of $Z G$ and $U_{1}$ the group of units of $\oplus t_{d} z_{d}$. Then $U_{1}$ is generated by the units of the components $z_{d}$ - and the unit group of each $z_{d}$ is finitely generated by Dirichlet's Unit Theorem (cf. [3] p. 124 Satz 100; we note that $Z_{d}$ is the ring of integers of $Q_{d}$-cf. [6], p. 264, 7-5-4 Theorem). Now we show that $U_{1} / U$ is finite. It is sufficient to show that every unit $u$ of $Z_{d}$ has finite order, mod $Z(G)$. Letting $\underline{\underline{A}}=$ principal ideal generated by $k$ in $z_{d}$, we have $u^{\phi(\underline{\underline{A}})} \equiv 1$ (mod $\left.\underline{\underline{A}}\right)$ where $\phi(\underline{\underline{A}})$ denotes the number of reduced residue classes, mod $\underline{\underline{A}}$. Hence $u^{\phi(\underline{A})}=1+\lambda k$ (cf. [3], Satz 84), where $\lambda \in z_{d}$ and so $u^{\phi(A)} \in Z G$ since $\left[\oplus t_{d} Z_{d}: Z G\right]=k$. Hence $U=T \times F$, where $T$ is finite and $F$ is free abelian of rank $=$ the torsion-free rank of $U_{1}$. In Theorem 2, we have determined $T$ so it remains to calculate the torsion-free rank of $U_{1}$.

By Dirichlet's Theorem if $d>2, z_{d}$ has $\frac{\phi(d)}{2}-1$ independent units 
of infinite order. Thus the torsion-free rank of $U_{1}$ is $\sum_{d{ }_{m}} t_{d}\left(\frac{\phi(d)}{2}-1\right)$. But $\sum_{d\rceil_{m}} t_{d}(d)=n$, and $\sum t_{d}=z=$ number of $d \neq 1, \neq 2$

cyclic subgroups of $G$ and $t_{2}=$ number of elements of order 2 (by Theorem 1). An easy calculation shows that

$$
\sum_{d \neq 1, \neq 2} t_{d}\left(\frac{\phi(d)}{2}-1\right)=\frac{1}{2}\left(n+1+t_{2}-22\right)
$$

4. The idempotents of $Q G$

In what follows we will identify $Q G$ with $\oplus t_{d} Q_{d}$ (using the isomorphism of Theorem 2). If $D$ is a subgroup of $G$ with $G / D$ cyclic, then $\pi_{D}$ will denote the projection of $Q G$ onto $Q_{d}$ whose associated character $X_{D}$ has kernel $D$. By Theorem 2, there is exactly one such projection. Thus if $u=\oplus s_{D}$, then $s_{D}=\pi_{D}(u)$. It is clear that $u$ is idempotent if, and only if, each $s_{D}$ is either 0 or 1 -i.e. each idempotent is a sum of primitive idempotents $u_{D}$, where $\pi_{D}\left(u_{D}\right)=1$ and $\pi_{D^{\prime}}\left(u_{D}\right)=0$ for $D^{\prime} \neq D$. In Theorem 5 we obtain the representation of the idempotents $u_{D}$ as elements of $Q G$.

THEOREM 5. FOP $D \leqq G$ with $G / D$ cyclic, let $u_{D} \in(Q G)$ be the primitive idempotent which corresponds to the identity of $\pi_{D}(Q G)$, and for $K \leqq G$ let $e_{K}=\sum_{G / D \text { cyclic }} u_{D} \cdot$ Then

$$
\begin{gathered}
e_{K}=\frac{1}{|K|} \sum_{k \in K} k \\
u_{D}=\sum_{D \leq H} \mu(|H / D|) e_{H},
\end{gathered}
$$

where $\mu$ is the Möbius $\mu$-function.

Proof. Consider the element $r_{K}=\frac{1}{|K|} \sum_{k \in K} k \in Q G$. For $H \leqq G$ with 
$G / H$ cyclic,

$$
\begin{aligned}
& \pi_{H}\left(r_{K}\right)=\frac{1}{|K|} \sum_{k \in K} \pi_{H}(k)=\frac{1}{|K|} \sum_{k \in K} x_{H}(k)= \begin{cases}1 & \text { if } K \leqq H, \\
0 & \text { otherwise }\end{cases} \\
& \pi_{H}\left(e_{K}\right)=\sum_{\substack{K \leq D \\
G / D \text { cyclic }}} \pi_{H}\left(u_{D}\right)= \begin{cases}1 & \text { if } K \leqq H, \\
0 & \text { otherwise. } .\end{cases}
\end{aligned}
$$

Hence $e_{K}=r_{K}=\frac{1}{|K|} \sum_{k \in K} k$, as claimed.

(12) follows from the Möbius inversion formula.l

COROLLARY. Let $G=(a)$ be cyclic of order $p^{n}$ (p a prime), and Zet

$$
\begin{gathered}
e_{i}=\frac{1}{p^{n-i}} p_{k=0}^{n-i}\left(a^{p^{i}}\right)^{k} \in Q G, 0 \leqq i \leqq n, \\
u_{i}=e_{i}-e_{i-1}, 1 \leqq i \leqq n .
\end{gathered}
$$

Then

$$
\begin{aligned}
& e_{i} \leftrightarrow 1 \oplus \ldots \oplus \oplus 1 \oplus 0 \oplus \ldots \oplus 0 \in \underbrace{\oplus}_{j=0} z^{j}, \\
& u_{i} \leftrightarrow 0 \oplus \ldots \oplus 1 \oplus \ldots \oplus 0 \in \sum_{j=0}^{n} z_{p^{j}} \cdot
\end{aligned}
$$

5. The index of $Z(G)$ in $t_{d} Z_{d}$

THEOREM 6. Let $G=(a)$ be a cyclic of order $p^{n}$ ( $p$ a prime) and let $a_{i}$ be defined by (13). Then the set $\left\{e_{i} a^{j}\right\}$

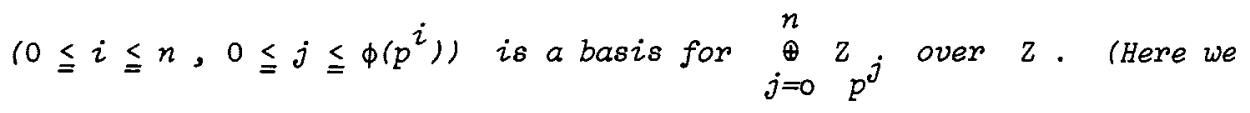

1 We are indebted to the referee for the use of the Möbius $\mu$-function in the representation of $u_{D}$ and for pointing out that the Möbius inversion formula holds under these circumstances. 
256

Raymond G. Ayoub and Christine Ayoub

are identifying $e_{i} a^{j}$ with its image in ${\underset{j=0}{\oplus} Z_{p^{j}}}^{\prime}$.

Proof. Let $M=\bmod _{Z}\left\{e_{i} a^{j}\right\} \underset{\left.\substack{0 \leq i \leq n \\ 0 \leq j \leq \emptyset}, i p^{i}\right)}{ }$. By the Corollary to Theorem

5, $M \leqq \underset{j=0}{\oplus} Z_{p^{j}}^{n}$ since $e_{i}$ and $a^{j} \in \underset{j=0}{\oplus} Z_{p^{j}}^{n}$. The number of generators $e_{i} \alpha^{j}$ is $\sum_{j=0}^{n} \phi\left(p^{j}\right)=p^{n}$. Thus if we establish that $M=\stackrel{\oplus}{j=0}_{p^{j}}^{n} z_{\text {, the }}$ theorem will be proved.

We prove by induction on $i$, that $e_{i} a^{j}$ and $u_{i} \alpha^{j}$ (where $u_{i}=e_{i}-e_{i-1}$, are in $M, \forall j \in N$. Note that

$$
a^{j}=1 \oplus \rho_{1}^{j} \oplus \ldots \oplus \rho_{i}^{j} \oplus \ldots \oplus \rho_{n}^{j},
$$

where $\rho_{i} \in 2_{p^{i}}$ is a primitive $p^{i}$-th root of unity (strictly speaking the $"="$ should be $" \leftrightarrow "$ but we are identifying $Z G$ with its image). If $i=0, e_{0} a^{j}=u_{0} a^{j}=e_{0} \in M, \forall j \in N$. So we assume that $e_{i-1} a^{j}$ and $u_{i-1} a^{j} \in M, \forall j \in N \cdot u_{i} a^{j}=0 \oplus \ldots \oplus \rho_{i}^{j} \oplus \ldots \oplus 0$ and $z\left(\rho_{i}\right)$ is generated over $z$ by $1, \rho_{i}, \rho_{i}^{2}, \ldots, \rho_{i}^{\phi}\left(p^{i}\right)-1$; hence $u_{i} a^{j} \in M, \forall j \in N$ if $u_{i} a^{j} \in M$ for $0 \leqq j \leqq \phi\left(p^{i}\right)$. So consider $u_{i} a^{j}$ with $0 \leqq j \leqq \phi\left(p^{i}\right)$. We have

$$
u_{i} a^{j}=e_{i} a^{j}-e_{i-1} a^{j}
$$

and $e_{i} a^{j} \in M$ since it is one of the generators of $M, e_{i-1} \alpha^{j} \in M$ by the induction assumption. Hence $u_{i} a^{j} \in M$. Also $e_{i}=u_{i}+e_{i-1}$ and hence

$$
e_{i} a^{j}=u_{i} a^{j}+e_{i-1} a^{j} \in M, \forall j \in N
$$

https://doi.org/10.1017/S0004972700041496 Published online by Cambridge University Press 
Clearly $\underset{j=0}{\oplus} Z_{p^{j}}$ is generated by $u_{i} a^{j} ;$ hence $\oplus Z_{p^{j}}^{n} \leqq M \leqq \oplus Z p^{j}$ so $M=\stackrel{\leftrightarrow}{\oplus} Z{ }_{j=0}^{j} p^{j}$

THEOREM 7. Let $G=(a)$ be cyclic of order $p^{n}$ ( $p$ a prime) and let $e_{i}(0 \leqq i \leqq n)$ be defined by (13). Then

(A) $e_{i} a^{j}$ has order $p^{n-i}, \bmod z G(\forall j \in N)$.

(B) The elements $e_{i} a^{j}\left(0 \leqq i \leqq n-1,0 \leqq j<\phi\left(p^{i}\right)\right)$ form a basis

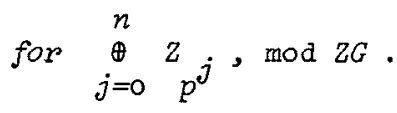

(C) $\bigoplus_{j=0}^{n} Z_{p^{j}}: Z G \mid=p^{1+p+\ldots+p^{n-1}}=p^{\left(p^{n}-1 / p-1\right)}$.

Proof. By the previous theorem, the $e_{i} a^{j} \quad\left(0 \leqq i \leqq n, 0 \leqq j<\phi\left(p^{i}\right)\right)$ form a basis for $\oplus_{j=0}^{n} Z_{p^{j}}=M$. Since $e_{n} a^{j}=a^{j} \in Z G$, the elements $e_{i} a^{j}\left(0 \leqq i \leqq n-1,0 \leqq j<\phi\left(p^{i}\right)\right)$ generate $M$, mod $2 G$. It is clear from the definition of $e_{i}$, that $e_{i} a^{j}$ has order $p^{n-i} \quad(\forall j \in N)$. Hence (A) holds. To prove (B), we need only show that the $e_{i} a^{j}\left(0 \leqq i \leqq n-1,0 \leqq j<\phi\left(p^{i}\right)\right)$ are linearly independent, mod $Z G$. We prove this by induction on $n$. If $n=1$, there is just one generator $e_{0}$ and so there is nothing to prove.

Now assume the result true in case of a cyclic group of order $p^{n-1}$. Let $B=(b)$, where $b=a^{p} ; B \leqq G$ has order $p^{n-1}$ and so if we let

$$
w_{i}=\frac{1}{p^{(n-1)-i}} p_{k=0}^{(n-i)-1-1}\left(b^{\left.p^{i}\right)^{k}},\right.
$$

the induction hypothesis implies that $w_{i} b^{j} \quad\left(0 \leqq i \leqq n-2,0 \leqq j<\phi\left(p^{i}\right)\right)$ are linearly independent, $\bmod Z B$. But 


$$
w_{i}=\frac{1}{p^{n-(i+1)}} \sum_{k=0}^{p^{n-(i+1)}-1}\left(a^{p^{i+1}}\right)^{k}=e_{i+1}
$$

and $w_{i} b^{j}=e_{i+1} a^{p j}$. Hence the elements $e_{i+1} a^{p j} 10 \leqq i \leqq n-1$, $0 \leqq j<\phi\left(p^{i}\right)$ ) are linearly independent, $\bmod Z B$.

Now assume

$$
\left.c_{00} e_{0}+\sum_{i=1}^{n}\left(\sum_{j=0}^{\phi\left(p^{i}\right)-1} c_{i j} e_{i} a^{j}\right)=t \in Z G \text { (where } c_{i j} \in Z\right) .
$$

Multiplying by $p^{n}$ we get:

$$
\begin{aligned}
p^{n} c_{00} e_{0}+p\left(\sum \sum c_{i j} p^{n-1} e_{i}\right)=p^{n} t & \Rightarrow p^{n} c_{00} e_{0} \in p Z G \\
& \Rightarrow c_{00}\left(\sum_{i=0}^{p^{n}-1} a^{i}\right) \in p 2 G \text { (using (18)) } \\
& \Rightarrow c_{00} \equiv 0(\bmod p) .
\end{aligned}
$$

So we can set $c_{00}=p c_{00}^{\prime}$ with $c_{00}^{\prime} \in Z$. Noticing that

$\frac{1}{p^{n-1}} \sum_{k=0}^{n} a^{k}=e_{1}+e_{1} a+\ldots+e_{1} a^{p-1}$, we can write (19) in the form

(20) $\quad c_{\infty 0}^{\prime}\left(e_{1}+\ldots+e_{1} a^{p-1}\right)+\sum_{i=1}^{n-1} \sum_{j=0}^{i} c_{i j}^{i} e^{i} a^{j}=t \in Z G$.

Consider now the terms involving only the $a^{j}$ with $j \equiv p-1(\bmod p)$. We get

$$
\begin{aligned}
& c_{00}^{\prime} e_{1} a^{p-1}+\sum_{i=2}^{n-1} \sum_{\substack{\leq j<\phi(p) \\
j \equiv p-1(\bmod p)}} c_{i j} e_{i} \alpha^{j} \\
& =c_{00}^{\prime} e_{1} a^{p-1}+\sum_{i=2}^{n-1} p_{i=0}^{i-1}-p^{i-2}-1 \quad c_{i, l p \rightarrow(p-1)} e_{i} a^{l p+(p-1)} \\
& =t^{\prime} a^{p-1} \text { with } t^{\prime} \in Z B \text {. }
\end{aligned}
$$

But as we saw above the $e_{i+1} a^{2 p}$ 
$\left(0 \leqq i \leqq n-1,0 \leqq 2 \leqq \phi\left(p^{i}\right)-1=p^{i}=p^{i-1}-1\right)$ are independent, mod $2 B$. Therefore $c_{\text {oO }}^{\prime} \equiv 0\left(\bmod p^{n-1}\right)$ and

$$
c_{i, \tau_{p+(p-1)}} \equiv 0 \quad\left(\bmod p^{n-i}\right) \text { for } 2 \leqq i \leqq n-1,0 \leqq 2<\phi\left(p^{i-1}\right) .
$$

Since $c_{00}=p c_{00}^{\prime}, c_{00} \equiv 0\left(\bmod p^{n}\right)$ and we obtain

$$
\sum_{i=1}^{n-1} \sum_{j=0}^{i} e_{i j} e^{i} \alpha^{j} \in Z G \text {. }
$$

Repeating the argument (with $p-1$ replaced by $s$ ) we obtain

$$
\begin{aligned}
c_{i, I_{p+s}} \equiv 0\left(\bmod p^{n-i}\right) \text { for: } 1 \leqq i \leqq n-1 \\
0 \leqq 2<\phi\left(p^{i-1}\right) \\
0 \leqq s \leqq p-1 .
\end{aligned}
$$

But if $0 \leqq j<\phi\left(p^{i}\right), j \equiv s(\bmod p)$ for some $s$ in the range $0 \leqq s \leqq p-1$, and $j=2 p+s$ with $0 \leqq z<\phi\left(p^{i-1}\right)$. Hence from (21) we deduce that

$$
c_{i, j} \equiv 0 \quad\left(\bmod p^{n-i}\right), 1 \leqq i \leqq n-1,0 \leqq j<\phi\left(p^{i}\right)
$$

and this is what we wanted to show. Thus $(B)$ is established.

Finally we consider (c). Since $e_{i} a^{j}$ has order $p^{n-i}$, mod $2 G$, and these elements form a basis for $\underset{j=0}{\oplus} Z^{j}=M$, mod $Z G$, the order of the quotient group $M / Z G$ is

$$
p^{n}\left(p^{n-1}\right)^{\phi(p)}\left(p^{n-2}\right)^{\phi\left(p^{2}\right)} \ldots(p)^{\phi\left(p^{n-1}\right)} .
$$

But since $n+(n-1) \phi(p)+\ldots+\phi\left(p^{n-1}\right)=1+p+\ldots+p^{n-1}$ (this can easily be established by induction), we have

$$
\left[\begin{array}{lll}
\oplus & 2 \\
j=0 & p^{j} & Z G
\end{array}\right]=p^{1+p+\ldots+p^{n-1}} .
$$

COROLLARY. Let $G=(a)$ be cyclic of order $p^{n}$. If we define 
$t_{i}=\sum_{k=0}^{n-i}\left(a^{p^{i}}\right)^{k} 0 \leqq i \leqq n$, the elements $t_{i} a^{j}$

$\left(0 \leqq i \leqq n, 0 \leqq j<\phi\left(p^{i}\right)\right)$ form a basis for $z G$ (over $z$ ).

Proof. Let

$$
\begin{aligned}
M_{1}=\operatorname{Mod}_{Z}\left\{t_{i} a^{j}\right\}_{0} & \leq i \leq n \\
0 & \leqq j<\phi\left(p^{i}\right) .
\end{aligned}
$$

Clearly $M_{1} \leqq z G<M=\underset{\substack{0 \leq i \leq n \\ 0}}{\oplus} Z\left(e_{i} a^{j}\right)$. Let $r \in Z G$; then $0 \leq i \leq n$
$0 \leq j<\phi \overline{7} p$

$r=\sum_{\substack{0 \leq i \leq n \\ 0 \leq j}} c_{i j} e_{i} a^{j}$ with $c_{i j} \in Z$ so that $c_{i j}=c_{i j}^{\prime} p^{n-i}$ with $0 \leq j<\phi\left(p^{i}\right)$

$c_{i j}^{\prime} \in Z$ since the $e_{i} \alpha^{j}$ are linearly independent, mod $Z G$. Thus $r=\sum c_{i j}^{\prime} t_{i} a^{j} \in M_{1}$, since $p^{n-i} e_{i}=t_{i}$. Hence $M_{1}=2 G$. But the $t_{i} \alpha^{j}$ are linearly independent since the $e_{i} a^{j}$ are; thus

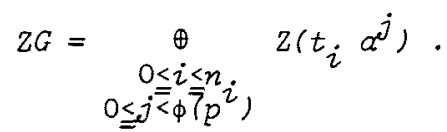

PROPOSITION 4. Let $B_{i} \leqq A_{i}$ be free abezian groups of rank $n_{i}$ and Let $A_{i} / B_{i}$ have elementary divisors $d_{i 1}, \ldots, d_{i n_{i}}(1 \leqq i \leqq r)$. Then $A_{1} \otimes \ldots \otimes A_{r / B_{1} \otimes \ldots \otimes B_{r}}$ has a basis of $\prod_{i=1}^{r} n_{i}$ elements whose orders are $d_{i j_{1}}, \ldots, d_{r j_{r}}$, where $1 \leqq j_{i} \leqq n_{i}$.

Proof. Let $A_{i}$ have a basis $a_{i 1}, \ldots, a_{i n_{i}}$ and $B_{i}$ a basis $b_{i 1}=d_{i 1} a_{i 1}, \ldots, b_{i n_{i}}=d_{i n_{i}} a_{i n_{i}}$ (for $\left.1 \leqq i \leqq r\right)$. Then $A_{1} \otimes A_{r}$ has a basis $a_{1 j_{1}} \otimes \ldots \otimes a_{r j_{p}}\left(1 \leqq j_{i} \leqq n_{i}\right)$ and $B_{1} \otimes \ldots \otimes B_{r}$ has a basis $b_{1 j_{1}} \otimes \ldots \otimes b_{r j_{r}}=a_{1 j_{1}} \ldots a_{r j_{r}}\left(a_{1 j_{1}} \otimes \ldots \otimes a_{r j_{r}}\right) \quad\left(1 \leqq j_{i} \leqq n_{i}\right)$ and the Proposition follows.

COROLLARY. Under the hypothesis of the Proposition, 
$\left[A_{1} \otimes \ldots \otimes A_{r}: B_{1} \otimes \ldots \otimes B_{r}\right]=\prod_{i=1}^{r}\left|\frac{A_{i}}{B_{i}}\right|^{j \neq i}{ }^{\operatorname{rank}\left(A_{j}\right)}$

If $G$ is a finite abelian group, and $G=C_{1} \times \ldots \times C_{k}$, where each $C_{i}$ is cyclic of prime power order, then $Z G=Z C_{1} \otimes \ldots \otimes Z C_{k}$ (note after Proposition 2) and for $1 \leqq i \leqq k, Z C$ is isomorphically imbedded in $\stackrel{\tau_{i}}{\oplus} z_{p_{i}}^{j=M_{i}}$, where $\left|C_{i}\right|=p_{i}^{\tau_{i}}$. Theorem 7 tells us the structure of the finite additive (abelian) group $M_{i} / \mathrm{ZC}$. Hence by using Proposition 4 we could calculate $\left[M_{1} \otimes \ldots \otimes M_{k}: 2 G\right]$. However, this does not give us the index of $Z G$ in $\underset{d \mid m}{\otimes} t_{d} Z_{d}$ since $M_{1} \otimes \ldots \otimes M_{k}$ is only imbedded in this last group as a subgroup of finite index. The calculation of this index would seem to be rather complicated.

\section{References}

[1] S.D. Berman, "On the equation $x^{m}=1$ in an integral group ring", Ukrain. Mat. $\breve{z}, 7$ (1955), 253-261.

[2] Charles W. Curtis and Irving Reiner, Representation theory of finite groups and associative algebras, (Interscience, New York, 1962).

[3] Erich Hecke, Vorlesungen über die Theorie der algebraischen Zahlen, (Chelsea Publ., New York, 1948).

[4] Graham Higman, "The units of group rings", Proc. London Math. Soc. (2), $46(1940), 231-248$.

[5] Sam Perlis and Gordon L. Walker, "Abelian group algebras of finite order", Trans. Amer. Math. Soc. 68 (1950), 420-426.

[6] Edwin Weiss, Algebraic number theory, (McGraw-Hill, New York, 1963).

Pennsylvania State University, University Park, Pennsylvania, USA. 